Ann. Abeilles I964, 7 (3), 20I-205.

\title{
ÉTUDE BACTÉRIOLOGIQUE D'UN RAYON DE COUVAIN D`ABEILLE SIMULANT LA LOQUE AMÉRICAINE
}

\author{
C. TOUMANOFF et G. ZARIFOPOULOS \\ Insitut Pasteur, Paris \\ Institut bactériologique vétérinaive d'Athènes (Grèce).
}

SOMMAIRE

Ayant examiné un vieux rayon de couvain, dont l'aspect, l'odeur et les spores bactériemes faisaient croire à la loque américaine, les auteurs ont isolé un autre germe que Bacillus larvae. Il s'agissait d'un bacille dont l'ensemble des caractères a permis de le classer comme Bacillus circulans JORDAN.

Dans des échantillons de vieux rayons ayant contenu du couvain mort, nous avons parfois constaté la présence d'écailles de larves ressemblant fortement à celles qui caractérisent les larves mortes de la loque américaine et prêtant facilement à confusion avec cette maladie. D'autre part, il se dégageait de ces rayons une odeur semblable à celle qui émane des larves mortes de loque maligne.

C'est ainsi qu'en I 962 nous avons reçu un vieux rayon de couvain d'une ruche dont la colonie a péri aut cours de l'année précédente. Ce rayon contenait plusieurs écailles desséchées et une larve décomposée et semi-gluante ; plusieurs cellules operculées étaient d'autre part percées par les Abeilles. L'odeur également se rapprochait de celle de la loque américaine. En procédant à l'examen microscopique, nous avons décelé dans les écailles ainsi que dans la larve des spores bactériennes.

On pouvait donc apparemment croire à la loque américaine et c'est sous cette désignation que ce rayon nous a été envoyé par un spécialiste apicole dont la compétence en la matière ne fait aucun doute, mais qui cependant était induit en erreur, n'ayant recours vraisemblablement qu'à un examen portant sur l'aspect du rayon, son odeur et sur la présence des spores.

Nous avons remarqué tout de suite que les spores étaient différentes de celles de Bacillus larvae et que les quelques rares bâtonnets présents dans le corps de la larve avaient un aspect différent de celui des formes végétatives de ce bacille quoique 
les bacilles dans un corps en voie de décomposition puissent revêtir des formes variées. İn l'occurrence, les bâtonnets étaient gros et déformés, mais néanmoins on pouvait penser qu'il s'agissait dans ce cas de la loque maligne, $B$. larvae présentant parfois des formes d'involution très variées.

C'est ainsi que l'isolement du germe était indispensable et, pour cela, nous avons eu recours à la gélose au jaune d'cuf qui est le nilieu de prédilection pour Bacillus larvae.

Nous avons alors constaté qu'il ne s'agissait pas de ce bacille, mais d'un autre germe sporulé dont nous présentons ci-dessous la description. Il s'agit d'un bacille Gram-négatif dont les dimensions varient de 0,6 à $5 \mu$ ayant des spores ellipsoïdales mesurant de 0,3 à $\mathrm{r}, 4 \mu$. On observe une certaine variation dans l'aspect des spores qui peuvent se présenter aussi bien sous l'aspect cylindrique que réniforme. Leur formation est sub-terminale ou parfois presque médiane.

La croissarce sur la gélose nutritive et la gélose sérum se fait sous forme d'un enduit plutôt sec. Sur le bouillon ordinaire, on note une croissance faible et sur la gélatine une absence de liquéfaction.

I a croissınce est légère sur la gélose nutritive au rouge neutre. Pas de virage ni de gaz. Sur pomme de terre : culture chagrinée. I,e lait tournesolé n'est pas modifié ; le fetit-lait to:arnesolé est décoloré, mais on n'observe pas le phénomène de caméléonage.

Parmi les glucides : le galactose et le glucose fermentent en 24 heures ; le maltose le lévulose, 1'arabinose et le lactose sont fermentés en 48 heures ; le xylose, 1'arabinose et le lactose donnent un virage en quatre jours. Pas d'action notable sur le saccharose, le dulcitol et le mannitex.

Réactions d'urée et indole : négatives.

Catalase : négative ; par contre, réaction nitrate-nitrite positive. I a production de l'acétyl-méthylcarbinol est négative. Réaction R M : positive. Pas de croissance sur le milieu de Simmons. Fn ce qui concerne l'amidon, il est hydrolysé, mais légèrement.

I a température optima de croissance est de $37^{\circ} \mathrm{C}$. On n'observe de croissance ni à des températures de o à $4^{\circ} \mathrm{C}$, ni à la température de $65^{\circ} \mathrm{C}$.

L'ensemble des caractères_de ce bacille nous 1'a fait classer comme Bacillus circulans JoRDAN.

Les spécialistes de bactériologie n'ignorent pas que $B$. circulans est largement répandu dans la nature et qu'on le trouve aussi bien dans l'eau que dans les poussières. On n'ignore pas non plus qu'il s'agit d'une espèce du genre Bacillus qui ne manifeste pas d'action pathogène certaine sur 1'homme, les animaux et entre autres les insectes.

Néanmoins HEINECKE (I956) a observé une septicémie due à Bacillus circulans chez Blatta orientalis. STEInhaus, à juger de la dernière publication de Heñecke, considère que $B$. circulans doit être classé comme un saprophyte sans importance. Cette opinion de STEINHAus est basée vraisemblablement sur le fait que l'infection des insectes per os par certains germes qui sont isolés au cours de leur septicémie s'avère inefficace.

Or, METArNIKOFF (I927) a signalé plusieurs cas de ce genre. Les bactéries qui provoquent une affection dans la nature sont inaptes, dans les conditions expérimentales, à produire la mềne maladie. 


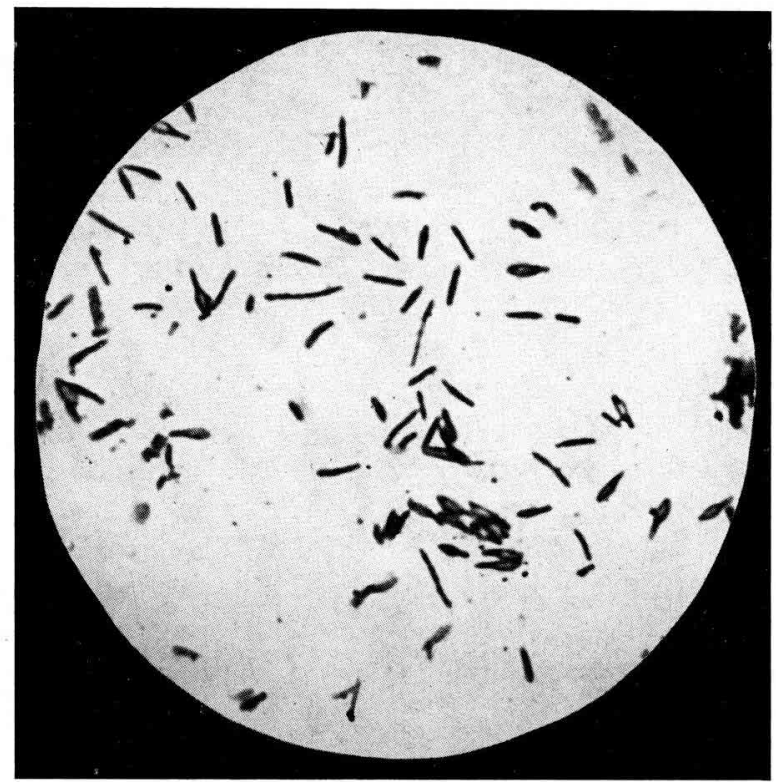

Photomicrographie dans nu frottis d'une culture de $\mathrm{B}$. circulans (sur gélose ordinaire) de 24 heures, montrant déjà la sporulation $\times 900)$
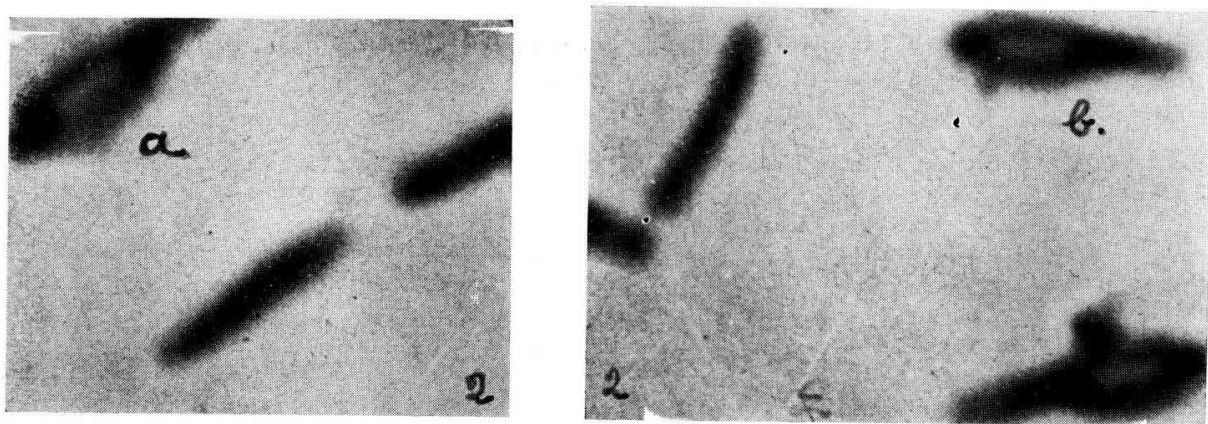

Le même bacille fortement grossi (environ $\times 6300)$. On y voit la formation des spores : a et b les modalités semblables à celles de $B$. larvae. 
C'est ainsi que la constatation de HEINECKE concernant la septicémie de Blatta orientalis due à $B$. circulans ne doit pas être réfutée.

Dans une récente communication, HEINECKE a fait connaître que le Bacillus circulans, considéré comme un saprophyte inoffensif, peut libérer une toxine, comparable à celle qui fut isolée par 1'un de nous, de Bacillus lavvae, l'agent pathogène de la loque américaine.

Il n'est pas à exclure ainsi que la présence de Bacillus circulans dans le rayon de couvain diagnostiqué comme une loque américaine n'est pas fortuite et qu'il s'agisse 1 à d'une infection qui ait pu se produire à la faveur de certaines circonstances difficiles à définir et qui n'est peut-être que rare, voire exceptionnelle.

On peut concevoir que c'est la détérioration de la membrane péritrophique qui est parfois la cause de la septicémie aussi bien chez les larves d'Abeilles que chez les Abeilles adultes. Cette détérioration peut être due à l'action des poisons et aussi à l'intoxication par certains produits alimentaires. In ce qui concerne les Abeilles, elle peut être due à l'ingestion de certains pollens. Les microorganismes ayant pénétré dans le tube digestif, ouvrent alors la voie à la septicémie.

En l'occurence, nous n'avons pas la prétention d'avoir découvert une nouvelle maladie des larves d'Abeilles, d'autant plus que nous avions affaire à un vieux rayon de couvain et que nous n'avons pas une preuve certaine, expérimentale, de l'effet pathogène de Bacillus circulans pour les larves d'Abeilles.

On ne doit pas cependant écarter l'existence de mortalités de ces larves dues à d'autres germes que ceux qui provoquent les maladies bien connues.

Afin que les connaissances dans ce sens progressent, il faudrait que dans les laboratoires où l'on pratique le diagnostic des maladies des Abeilles et de leur couvain on ait recours constamment à l'examen bactériologique approfondi.

L'un de nous (ToumanofF, I927), a déjà signalé un cas aberrant d'infection du couvain d'Abeilles ressemblant à la loque européenne et accompagnée d'une microflore spéciale qui a été décrite. Ce fait a été confirmé par MorIsON (I929) en Écosse, mais est resté inédit.

On doit dire que dans certains laboratoires on classe parfois les maladies d'après l'aspect extérieur du couvain, son odeur et l'examen microscopique superficiel qui dénote, soit la présence de spores, soit celle de formes végétatives de bactéries ressemblant à celles de la loque européenne ou américaine.

Ces procédés nous paraissent insuffisants et n'offrent pas la garantie d'un diagnostic certain. Il faut, il nous semble, procéder à l'isolement et l'étude de la flore bactérienne.

Reçu pour publication en juin $\mathrm{1} 964$.

\section{SUMMARY}

A BACTERIOLOGICAL STUDY OF A SHELF OF A BEE BROOD COMB IN A CONDITION RESEMBLING AMERICAN FOULIBROOD

The authors isolated from an old brood comb shelf which had the look, smell and bacterial spores similar to American foulbrood, an organism which was not Bacillus larvae. This bacillus was Gram negative and had ellipsoid spores. From its characteristics it was classified as Bacillus circulans 
Jordan. It was possible that the septicenia caused by $B$. circulans was sometimes due to a deterioration of the peritrophic membrane both in the larve and adult bee, and that this deterioration was due to the ingestion of certain pollens.

\section{RÉFÉRENCES BIBLIOGRAPHIQUES}

HeINECkE H., I956. Lber einch pathogenen Sporenbildner in der Ilaemolymphe von Blatia orientalis I, Zbl. Bakl, II, 109, 524-535.

HeINecke II., Ig62. Infektionversuche an Achroea grisella und Galleria mellonella. Zbl. Bakt II, 115.

IIETalikof S., 1927 . L'infection microbienne de l'Immunité chez la mite des Rbeilles. Monographie de l'Insitut Pasteur, L'aris, r.to p.

Metalnikoff S. et Chorine V., igzg. I'tilisation des microbes dans la lutte contre la Pyrale de mais (Pyraush mubilalis IU⿴囗N) Ann. Inst. Pasteur, 43, $\mathrm{1} 39^{1-5}$.

Toumnof C., гg27. La flore microbienne d'un couvain malade. Recueil de Médecine vétérinaire, de l'École d. 1 llot. $103(22)$,

Toumixof C., 1950. Action de Bacillus larnae W. agent pathogène de la loque maligne des larves d'Abeilles sur les chenilles de Calleria mellonella L. C. R. Acad. Sci, 230, i 709.

Toumanorf C., I95. Les maladies des Abeilles. No spécial de la Rex. prans. Apiculture (325), i 3 , fig.

Stennaus E. A. et Jerrel E. A., I954. Further observations on Bacillas thuringiensis BerLiner and other sporeforming bacteria. Hilgutita, 23, $1-21$. 